\title{
The Impact of Maternal Obesity on Maternal and Fetal Health
}

Japmeet Sandhu, OMS III

The average person today eats more and moves less. The worldwide prevalence of obesity has increased over the past few decades. According to the CDC, in the US alone, $42.4 \%$ of adults are obese. Obesity is a significant public health concern that can lead to severe heart disease or diabetes. Obesity not only affects the mother, but as the rates of maternal obesity increase, it affects the fetus as well.

Obesity during pregnancy can have fatal consequences, with more than $50 \%$ of mortality cases during pregnancy, childbirth, or puerperium in women who are overweight or obese. Over $50 \%$ of women of childbearing age are overweight (BMI 25-29.9) or obese (BMI >30). 15\% of women start pregnancy obese, while $20-40 \%$ suffer from excessive weight gain during pregnancy. (1). Risks for both the mother and baby increase linearly with BMI. Risks for the mother include gestational diabetes, preeclampsia, postpartum endometritis, laceration episiotomy infection, cesarean delivery, postpartum hemorrhage, and the development of heart disease, diabetes or hypertension later on in life. Risks for the baby include stillbirth, congenital abnormalities, preterm birth, macrosomia, spontaneous abortion, and the development of obesity, diabetes, or heart disease later on in life. (2)

"PRAMS is the Pregnancy Risk

Assessment Monitoring System, and

it is an ongoing population-based

surveillance system that examines

trends in pre-pregnancy obesity by

maternal demographics and behavioral

characteristics."

PRAMS is the Pregnancy Risk Assessment Monitoring System, and it is an ongoing population-based surveillance system that examines trends in pre-pregnancy obesity by maternal demographics and behavioral characteristics. They collect self-reported data from maternal questionnaires on behaviors associated with pregnancy. Patterns reported from a 2003 study show that only 1 in 3 women had weight gain consistent with the Institute of Medicine recommendations and demonstrate trends in racial factors affecting weight gain. For instance, African American women are more likely to be overweight prior to pregnancy and more likely to gain weight above IOM guidelines. White females were more likely to report target weight gain, while Hispanic women were less likely to report target weight gains. Asian women were more likely to gain less than the recommended weight. (3). Another study brought in socioeconomic factors as well. They found that mothers who attended college were more likely to have normal pre-pregnancy BMI than those who did not. Also, mothers who had less money had a higher percentage of being obese pre-pregnancy. (4).

Recommendations for Weight Gain in Pregnancy

Body Mass Index

Recommended Weight Gain

$18.5-24.9 \mathrm{~kg} / \mathrm{m}^{2}$ (normal weight) $25-35 \mathrm{lb}(11.2-15.9 \mathrm{~kg}$ )

$25-29.9 \mathrm{~kg} / \mathrm{m}^{2}$ (overweight)

$15-25 \mathrm{lb}(6.8-11.2 \mathrm{~kg})$

$>30 \mathrm{~kg} / \mathrm{m}^{2}$ (obese)

$15 \mathrm{lb}(6.8 \mathrm{~kg})$

The impact of maternal obesity on maternal and fetal health. (3)

"Normal weight and obese women tend to gain the same amount of fat during pregnancy; however, fat in a normal weight woman accumulates in the peripheral body while fat in an obese woman accumulates in the abdominal area, liver, and placenta."

Less gestational weight gain (GWG) is recommended as the BMI increases. Studies showed that in obese women, less GWG is associated with an increased risk of preterm birth and having a small for gestational age (SGA) infant; however, less GWG does reduce the risk of macrosomia, hypertensive diseases of pregnancy, and cesarean delivery. (5) Guidelines today state that if obese women gain less weight than the IOM recommends but fetal growth is adequate, they do not need to increase their weight gain. It is all about finding a happy medium regarding GWG. However, if a woman has excessive GWG, then it is associated with an increased risk of obesity for the neonate later on in life. The risk for this is highest in the first trimester of pregnancy. Excessive weight gain in the second or third trimester is more associated with an increased risk of having a large for gestational age infant. (6).

Normal weight and obese women tend to gain the same amount of fat during pregnancy; however, fat in a normal weight woman accumulates in the peripheral body while fat in an obese woman accumulates in the abdominal area, liver, and placenta. Body fat

NEONATOLOGY TODAY is interested in publishing manuscripts from Neonatologists, Fellows, NNPs and those involved in caring for neonates on case studies, research results, hospital news, meeting announcements, and other pertinent topics.

Please submit your manuscript to: LomaLindaPublishingCompany@gmail.com 
distribution in obese women favors lipotoxicity and metabolic dysfunctions that expose the fetus to glucose and fat overload. This excess increases fetal size, fat mass and causes inflammation and metabolic dysfunction. (6). Obese women also have nutritional deficits caused by low-quality diets or dietary behavior. An imbalance of prenatal micronutrients increases the risk of delayed neurodevelopment. One study even showed that maternal prepregnancy obesity was associated with lower child IQ than the IQ of children born to normal-weight moms. (7).

\section{"Obese women have increased risks of complications at the time of labor and delivery. The rate of successful vaginal delivery decreases as maternal BMI increases."}

Obese women have increased risks of complications at the time of labor and delivery. The rate of successful vaginal delivery decreases as maternal BMI increases. The $\mathrm{C}$ section delivery rate for women < $200 \mathrm{lbs}$ was $18 \%$ compared to $39.6 \%$ in women > 200 lbs. (3) Not only are obese women more likely to have a C section, but they are also more likely to have a lower success rate of attempted vaginal birth after cesarean (VBAC). The VBAC success rate in women $<200$ lbs was $81.8 \%, 57.1 \%$ in women $200-300 \mathrm{lbs}$ but only $13.3 \%$ in women $>300 \mathrm{lbs}$. (3).

The risk for obesity in offspring starts with obesity at conception. Animal studies showed that offspring born from gametes of obese mothers and fathers that were fertilized in vitro and implanted in non-obese surrogates still had a higher risk of developing obesity and insulin resistance later in life. (6). Thus, not only does obesity affect the mother, but the fetus as well. Evidence suggests that genes and the environment both play a role in developing obesity. The first effects of the environment take place in the womb, where the fetus is entirely dependent on the mother, with the placenta and amniotic fluid being a source of microbial transmission from the mother's microbiome. Interestingly, maternal gut microbiota varies between normal weight and obese pregnant women. Obese women have an increase in Staphylococcus and E. Coli, increasing placental inflammation and insulin resistance in the fetus. (6). Obese women are also in a chronic state of inflammation with changes in the homeostasis of cytokines and adiponectin. Adiponectin allows for the placental transfer of nutrients and placental insulin-stimulated amino acid uptake. Obese women have low levels of adiponectin, which leads to changes in placental function and increased fetal growth. In addition, obese women have high plasma levels of glucose and free fatty acids, increasing the placental transfer of metabolites stored as fetal fat deposits. Maternal obesity also causes a reduction in placental villous proliferation and apoptosis, leading to an increase in stillbirths and a decrease in birth weight and neonatal survival. (1)

Maternal obesity is associated with abnormal fetal growth. Heavy women are less likely to have a pregnancy complicated by SGA or IUGR, but this protective effect disappears once maternal BMI $>30$. (3). There is no direct association between obesity and preterm birth. There is a slightly reduced risk of preterm birth in obese women because they exhibit less uterine activity than normal. However, obese women do have an increased overall preterm delivery rate due to medical complications that rise as BMI rises. (1). While obesity is generally associated with prolonged pregnancy, there is an increase in spontaneous extremely preterm labor (<28 weeks gestation) due to the increased risks of intrauterine bacterial infection, chorioamnionitis, and increased inflammatory markers. (5). On the other side, obese women have an $18-26 \%$ increased chance of delivering LGA infants, even if maternal diabetes is under control. (3). Excess fetal growth comes with more severe risks. These infants have a higher chance of unexplained intrauterine fetal death. They have an increased risk of shoulder dystocia or brachial plexus injury and a higher risk for fetal hypoxemia. Infants born to obese mothers usually have lower APGAR scores and an increase in NICU admission with complications such as neonatal trauma or incubator requirement. (3)

One of the most severe consequences of maternal obesity is a stillbirth. There is a consistent increase in the risk of stillbirth among obese women of all gestational ages. However, stillbirth and other complications can be reduced by timing the delivery. The lowest rates of neonatal death and cerebral palsy are associated with delivery at 39 weeks. The lowest rates of intrauterine demise and brachial plexus injury are by delivering even earlier. It is actually suggested that the optimal gestation age of delivery for obese women may be 39 weeks. Inducing labor at 39 weeks decreases the risk of $\mathrm{C}$ section in obese women without increasing the risk of adverse outcomes such as operative vaginal delivery, lacerations, and neonatal RDS. For appropriate candidates, labor induction at 38-39 weeks is currently preferred to elective $\mathrm{C}$ section. (5).

\section{"If an obese woman does become pregnant, a physician must carefully screen for maternal and fetal complications. They should recognize the increased risk of stillbirths and consider the risks and benefits of labor induction at} 38-39 weeks."

Another way to decrease risks is bariatric surgery. If before a planned pregnancy, it can reduce obesity-related complications for both the mother and child. Children born to a mother after bariatric surgery have a less chance of developing obesity than their siblings born prior to the surgery. Women are also less likely to develop GDM, hypertensive diseases of pregnancy, postpartum hemorrhage, and fetal macrosomia. However, the risks of preterm birth and SGA of the newborn are increased; thus, the current recommendations are waiting 12-24 months after surgery before conceiving. (5). Another beneficial action for obese mothers is breastfeeding. Breastfeeding is associated with improved cardiovascular risk, reduced risk of future DM2, and decreased visceral adiposity later in life. However, women with obesity are less likely to breastfeed. They have a delayed onset of milk production, a higher prevalence of insufficient breast glandular tissue, and reduced breastfeeding confidence. (5) It is important to not only pro- 
mote breastfeeding but help obese women feel confident and able to breastfeed.

With obesity on the rise, physicians need to discuss pregnancy plans with obese women well in advance of conception to ensure that medical comorbidities and medications are under control and optimized. Physicians should promote weight management to improve maternal and fetal health. If an obese woman does become pregnant, a physician must carefully screen for maternal and fetal complications. They should recognize the increased risk of stillbirths and consider the risks and benefits of labor induction at 38-39 weeks. Even after pregnancy, physicians should encourage obese women to reduce postpartum weight retention. As the incidence of maternal obesity increases, physicians must intervene and help prevent these sometimes fatal consequences.

\section{References:}

1. Tenenbaum-Gavish K, Hod M. Impact of maternal obesity on fetal health. Fetal Diagn Ther. 2013;34(1):1-7. Epub 2013/06/19. doi: 10.1159/000350170. PubMed PMID: 23774085.

2. Hashmi A, Soomro J, Iqbal Z, Soomro T, Saleem K. Maternal Obesity: A Global Health Problem and It's Implications on Maternal and Fetal Health. Journal of Family and Reproductive Health. 2010;4(4):155-60. PubMed PMID: 79424355.

3. Leddy MA, Power ML, Schulkin J. The impact of maternal obesity on maternal and fetal health. Rev Obstet Gynecol. 2008;1(4):170-8. Epub 2009/01/29. PubMed PMID: 19173021; PubMed Central PMCID: PMCPMC2621047.

4. Huang L, Liu J, Feng L, Chen Y, Zhang J, Wang W. Maternal prepregnancy obesity is associated with higher risk of placental pathological lesions. Placenta. 2014;35(8):5639. Epub 2014/06/17. doi: 10.1016/j.placenta.2014.05.006. PubMed PMID: 24930988.

5. Dutton $H$, Borengasser SJ, Gaudet LM, Barbour LA, Keely EJ. Obesity in Pregnancy: Optimizing Outcomes for Mom and Baby. Med Clin North Am. 2018;102(1):87106. Epub 2017/11/21. doi: 10.1016/j.mcna.2017.08.008. PubMed PMID: 29156189; PubMed Central PMCID: PMCPMC6016082.

6. Maffeis C, Morandi A. Effect of Maternal Obesity on Foetal Growth and Metabolic Health of the Offspring. Obes Facts. 2017;10(2):112-7. Epub 2017/04/07. doi: 10.1159/000456668. PubMed PMID: 28384625; PubMed Central PMCID: PMCPMC5644955.

7. Huang L, Yu X, Keim S, Li L, Zhang L, Zhang J. Maternal prepregnancy obesity and child neurodevelopment in the Collaborative Perinatal Project. Int J Epidemiol. 2014;43(3):78392. Epub 2014/02/27. doi: 10.1093/ije/dyu030. PubMed PMID: 24569381.
Disclosure Statement: The author has no relevant conflicts of interest to declare.
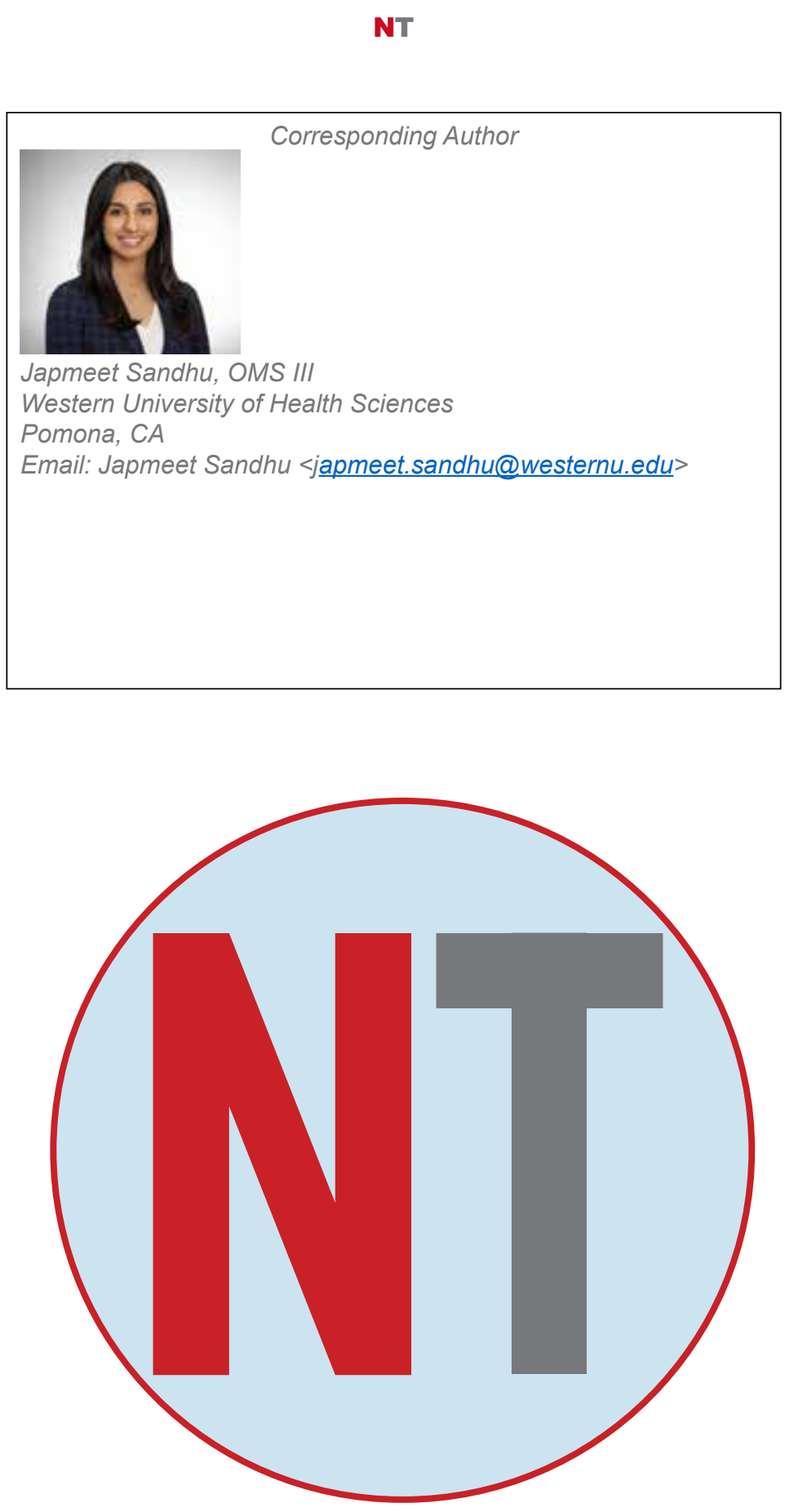

NEONATOLOGY TODAY is interested in publishing manuscripts from Neonatologists, Fellows, NNPs and those involved in caring for neonates on case studies, research results, hospital news, meeting announcements, and other pertinent topics. Please submit your manuscript to: LomaLindaPublishingCompany@gmail.com 